\title{
Correction to: Prophylactic sivelestat for esophagectomy and in-hospital mortality: a propensity score-matched analysis of claims database
}

\author{
Chikashi Takeda $^{1,2} \cdot$ Masato Takeuchi $^{1} \cdot$ Yohei Kawasaki $^{1,3} \cdot$ Hiroshi Yonekura $^{1} \cdot$ Isao Nahara ${ }^{1} \cdot$ Aki Kuwauchi $^{1}$. \\ Satomi Yoshida ${ }^{1}$. Shiro Tanaka ${ }^{1,4} \cdot$ Koji Kawakami $^{1}$
}

Published online: 14 January 2020

(c) Japanese Society of Anesthesiologists 2020

\section{Correction to: Journal of Anesthesia (2019) 33:230-237 https://doi.org/10.1007/s00540-018-2602-9}

In the original version of the article, the Tables 2 and 3 was published incorrectly. The correct version of Tables 2 and 3 are given below.

The original article can be found online at https://doi.org/10.1007/ s00540-018-2602-9.

Koji Kawakami

kawakami.koji.4e@kyoto-u.ac.jp

1 Department of Pharmacoepidemiology, Graduate School of Medicine and Public Health, Kyoto University,

Yoshida-Konoe-cho, Sakyo-ku, Kyoto 606-8501, Japan

2 Department of Anesthesia, Graduate School of Medicine, Kyoto University, Kyoto, Japan

3 Biostatistics Section, Clinical Research Center, Chiba University Hospital, Chiba, Japan

4 Department of Clinical Biostatistics/Clinical Biostatistics Course, Graduate School of Medicine, Kyoto University, Kyoto, Japan 
Table 2 Outcomes after propensity score adjustment

\begin{tabular}{|c|c|c|c|c|c|c|}
\hline & \multirow{2}{*}{$\begin{array}{l}\text { Sivelestat } \\
n=621\end{array}$} & \multirow{2}{*}{$\begin{array}{l}\text { Control } \\
n=2770\end{array}$} & \multicolumn{2}{|l|}{ Unadjusted analysis } & \multicolumn{2}{|c|}{$\begin{array}{l}\text { Propensity score-adjusted } \\
\text { analysis }\end{array}$} \\
\hline & & & OR $(95 \% \mathrm{CI})$ & $P$ value & $\mathrm{aOR}(95 \% \mathrm{CI})$ & $P$ value \\
\hline Hospital mortality & $33(5.3)$ & $81(2.9)$ & $1.86(1.23-2.82)$ & 0.0028 & $1.65(0.95$ to 2.88$)$ & 0.0772 \\
\hline ARDS & $10(1.6)$ & $30(1.1)$ & $1.49(0.73-3.07)$ & 0.2714 & $1.25(0.49$ to 3.17$)$ & 0.6380 \\
\hline Respiratory complication & $114(18.4)$ & $366(13.2)$ & $1.48(1.17-1.86)$ & 0.0009 & $1.24(0.91$ to 1.67$)$ & 0.1682 \\
\hline \multirow[t]{2}{*}{ Sepsis } & $31(5.0)$ & $95(3.4)$ & $1.48(0.98-2.24)$ & 0.0628 & $1.15(0.68$ to 1.95$)$ & 0.5933 \\
\hline & Mean \pm SE & Mean \pm SE & Difference $(95 \% \mathrm{CI})$ & $P$ value & Difference $(95 \% \mathrm{CI})$ & $P$ value \\
\hline Postoperative length of hospital stay & $45.0 \pm 1.9$ & $37.0 \pm 0.7$ & $8.1(4.1-12.0)$ & $<0.0001$ & $2.5(-2.3$ to 7.2$)$ & 0.3065 \\
\hline
\end{tabular}

$a O R$ adjusted odds ratio, $A R D S$ acute respiratory distress syndrome, $C I$ confidential interval, $S E$ standard error

Table 3 Outcomes after propensity score adjustment

\begin{tabular}{|c|c|c|}
\hline \multirow[t]{2}{*}{ Sensitivity analysis 1} & \multicolumn{2}{|c|}{ Propensity score-adjusted analysis } \\
\hline & $\mathrm{aOR}(95 \% \mathrm{CI})$ & $P$ value \\
\hline In-hospital mortality & $1.59(0.99$ to 2.58$)$ & 0.0578 \\
\hline ARDS & $1.65(0.70$ to 3.91$)$ & 0.2561 \\
\hline Respiratory complications & $1.24(0.96$ to 1.61$)$ & 0.1064 \\
\hline \multirow[t]{2}{*}{ Sepsis } & $1.28(0.79$ to 2.07$)$ & 0.3167 \\
\hline & Difference $(95 \% \mathrm{CI})$ & $P$ value \\
\hline Postoperative length of hospital stay & $3.1(-0.3$ to 6.5$)$ & 0.0769 \\
\hline Sensitivity analysis 2 & $\mathrm{aOR}(95 \% \mathrm{CI})$ & $P$ value \\
\hline In-hospital mortality & $1.24(0.73$ to 2.10$)$ & 0.4236 \\
\hline ARDS & $2.67(0.71$ to 10.1$)$ & 0.1474 \\
\hline Respiratory complications & $1.28(0.95$ to 1.73$)$ & 0.1092 \\
\hline \multirow[t]{2}{*}{ Sepsis } & $1.53(0.80$ to 2.94$)$ & 0.1978 \\
\hline & Difference $(95 \% \mathrm{CI})$ & $P$ value \\
\hline Postoperative length of hospital stay & $5.1(0.4$ to 9.9$)$ & 0.0348 \\
\hline Sensitivity analysis 3 & $\mathrm{aOR}(95 \% \mathrm{CI})$ & $P$ value \\
\hline In-hospital mortality & $1.38(0.72$ to 2.62$)$ & 0.3324 \\
\hline ARDS & $1.17(0.39$ to 3.47$)$ & 0.7817 \\
\hline Respiratory complications & $1.32(0.95$ to 1.83$)$ & 0.0989 \\
\hline \multirow[t]{2}{*}{ Sepsis } & $1.32(0.73$ to 2.39$)$ & 0.3672 \\
\hline & Difference (95\% CI) & $P$ value \\
\hline Postoperative length of hospital stay & $0.4(-3.8$ to 4.5$)$ & 0.8674 \\
\hline
\end{tabular}

$A R D S$ acute respiratory distress syndrome, $a O R$ adjusted odds ratio, $C I$ confidential interval, $S E$ standard error

Publisher's Note Springer Nature remains neutral with regard to jurisdictional claims in published maps and institutional affiliations. 